Klaus Miehling (Freiburg i. Br.)

\title{
Metrische Metronomangaben bei Max Reger?
}

Die sogenannte metrische Tempotheorie, wonach historische Pendel- und Metronomangaben als Doppelschläge zu interpretieren sind, wurde erstmals 1974 von Erich Schwandt zur Diskussion gestellt. ${ }^{1}$ Hatte sich dieser noch auf eine einzige Quelle, L'Affilards Principes trèsfaciles ( $\left.{ }^{5} 1705\right)$, beschränkt, so wurde das Prinzip bald auch auf andere Pendelangaben und auf die frühen Metronomangaben z. B. Beethovens übertragen und schließlich bis weit ins 19. Jahrhundert ausgedehnt. Nun glaubt Henrico Stewen in seiner Dissertation, metrische Metronombezeichnungen noch im 20. Jahrhundert bei den frühen Orgelwerken Max Regers ausmachen zu können. ${ }^{2}$ In der neuen Reger-Werkausgabe wurde diese Hypothese abgelehnt, ${ }^{3}$ in Veröffentlichungen von Daniel Stickan ${ }^{4}$ und Guy Bovet ${ }^{5}$ hingegen zustimmend aufgegriffen.

Der Befund, der Stewens Hypothese zugrunde liegt, unterscheidet sich allerdings von den Indizien, die bisherige Autoren für die metrische Theorie geltend machten: Offenbar hat hier der Komponist Reger Metronomangaben seines Herausgebers Karl Straube akzeptiert, die tatsächlich nur etwa halb so schnell wie seine ursprünglichen eigenen sind.

Die Metronomangaben zu Regers opp. 59 und 80

Bei den von Stewen diskutierten Metronomangaben handelt es sich um Gloria und Benedictus op. 59 Nr. 8 und 9, von Straube 1912 herausgegeben, sowie um die Fuge op.59/6 und die Fughetta op. 80/2, die Straube erst 1919, also nach Regers Tod, herausgab.

1 Erich Schwandt, „L'Affilard on the French Court Dances“, in: MQ, 60 (1974), S. 389-400.

2 Henrico Stewen, The Straube Code. Deciphering the Metronome Marks in Max Reger's Organ Music (= Sibelius Academy, Church Music Department Publications 30), Helsinki 2008.

3 Alexander Becker et al., Reger-Werkausg. I/2, Stuttgart 2011, DVD, „Zur Tempowahl bei Regers Werken“ (6): „Eher abwegig, zumal nicht belegbar, mutet in diesem Zusammenhang die doch etwas reißerisch beworbene (,The Straube Code'), zumindest für schnelle Sätze aufgestellte These des niederländischen Organisten Henrico Stewen an, ,Reger used double beat-metronome notation until about 1908‘ das Tempo müsste demnach halbiert werden - eine zweifelhafte Empfehlung, die ja bereits Hans Klotz in der Gesamtausgabe ausgesprochen hatte [...]." Allerdings bezieht sich Stewens These auch auf langsame Sätze.

4 Daniel Stickan, Tempofrage und Registrierkunst in Regers Orgelwerken und die Aufführungspraxis unter Reger, Straube und Straubes Schülern, <http://stickan.org/images/Reger-Essay-DS.pdf>, 29.7.2012.

5 Guy Bovet, „... und so schreibt man Geschichte ... (Lehrreiche Lektüren über jahrzehntealte Orgellegenden)“, in: Manfred Novak (Hrsg.), Theorien des Planyversums, Peter Planyavsky zum 65. Geburtstag, Münster 2012, S. 63-66. 


\section{Gloria in excelsis.}

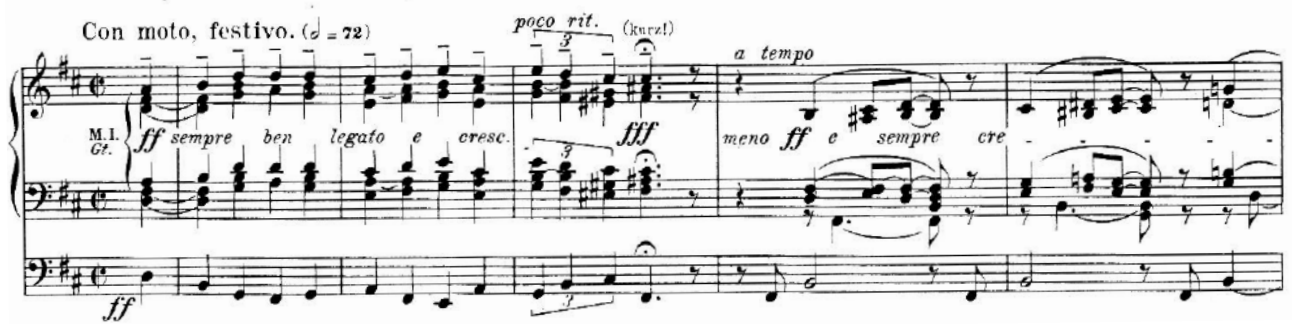

Nb 1: Gloria op. 59/8 (Ed. Peters 8758)

Das Gloria op. 59/8 beginnt nach Reger „Con moto, festivo“ mit $d=72$; Straube macht daraus "Maestoso“, • = 80. Auch die folgenden, vom Grundtempo nur um wenige Metronomstriche abweichenden Angaben, sind bei Straube ungefähr halbiert. Dessen Änderung der Vortragsbezeichnung weist auf eine langsamere Tempoauffassung, wenn auch das Ausmaß erstaunlich ist.

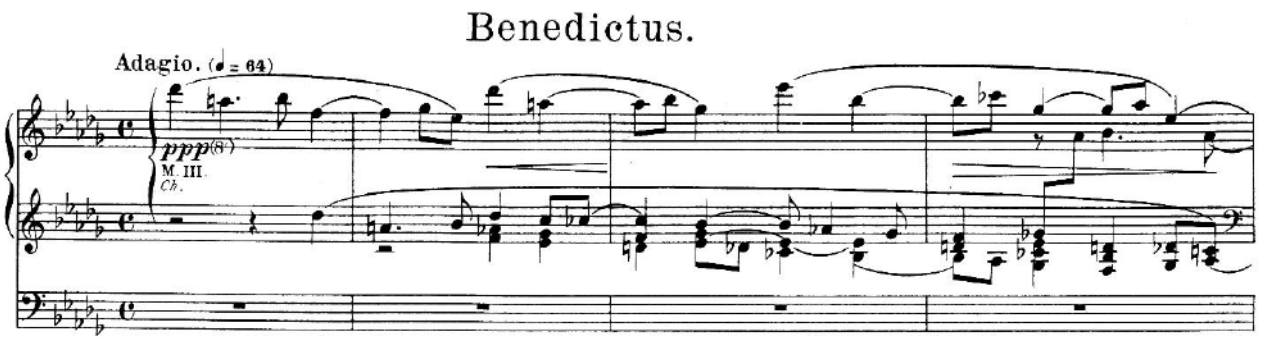

Nb 2: Benedictus op. 59/9 (Ed. Peters 8758)

Vom Benedictus op. 59/9 gibt es eine Fassung für Harmonium von 1908, die Stewen als Beleg für den angeblichen Übergang Regers von der metrischen zur mathematischen Notation um dieses Jahr herum verwendet. Diese Fassung zeige „beide Notationen. Wenn man diese Version mit der Orgelversion vergleicht, sind die Zahlen für den Anfang und Schluss die gleiche[n], während die Zahlen in der Mitte halbiert worden sind [...]."6 Ergänzen lassen sich diese Angaben durch eine Einspielung, die Reger 1913 für Welte-Mignon-Orgelrollen vorgenommen hat: ${ }^{7}$

6 Henrico Stewen, „Metronomzahlen bei César Franck und Max Reger“, in: Internationales TempoGiusto-Symposion 2009, Kongressberichte [sic], eigene Seitenzählung, S. 2, <http://www.wellermusik. de/Internationales_Tempo-Giusto_Symposion_2009_Kongressbericht.pdf>, 29.7.2012. Es handelt sich quasi um eine deutschsprachige Zusammenfassung der Dissertation. Stewens Bemerkungen zu César Franck beschränken sich auf das Zitieren der seiner Meinung nach zu schnellen Metronomangaben.

7 Vgl. die LP Max Reger spielt eigene Orgelwerke, EMI Electrola C 053-28925. 


\begin{tabular}{|c|c|c|c|c|}
\hline ab Takt & $\begin{array}{l}\text { Orgel } 1901 \text { (ed. 08) } \\
\text { (Reger) }\end{array}$ & $\begin{array}{l}\text { Harmonium } 1908 \\
\text { (Reger) }\end{array}$ & $\begin{array}{l}\text { Orgel } 1912 \\
\text { (Straube) }\end{array}$ & $\begin{array}{l}\text { Interpretation } 1913 \\
\text { (Reger) }\end{array}$ \\
\hline 1 & $\cdot=64$ (Adagio) & $\cdot=64$ (Adagio) & D. $=72$ (Adagio) & S $=72$ \\
\hline 24 & $d=96$ (Vivace assai) & • = 96 (Più mosso) & $\begin{array}{l}\cdot=72-92 \\
\text { (Un poco mosso) }\end{array}$ & $\cdot=67$ \\
\hline 41 & $d=130$ (Più vivace) & • = 144 (Più vivace) & - & $d=69$ \\
\hline 45 & - & - & $\begin{array}{l}\cdot=92 \\
\text { (Sostenuto molto) }\end{array}$ & $\cdot=53$ \\
\hline 50 & $\cdot=64$ (Adagio) & $\cdot=64$ (Adagio) & Tempo I (Adagio) & . $=64$ \\
\hline
\end{tabular}

Straubes Tempi werden durch Regers Interpretation weitgehend bestätigt. Wenn Reger teilweise sogar noch darunter bleibt, so liegt es an Ritenuti und Ritardandi, welche die Durchschnittswerte senken. Beweist das aber, dass die Adagiotempi der Harmoniumfassung metrisch gemeint sind? Dagegen spricht zunächst, dass der Änderung der Metronomangaben eine Änderung der Vortragsbezeichnungen entspricht (wie wir das auch beim Gloria beobachten konnten): Von „Adagio“ zu „Vivace assai“ der Orgelfassung befindet sich offensichtlich ein sehr großer Temposprung, der gut zu den Metronombezeichnungen passt. Das „Più mosso" der Harmoniumfassung dagegen impliziert nur eine graduelle Beschleunigung, die mit • = 96 immerhin fast $50 \%$ beträgt. Auch wenn solche Modifikationen bei Reger sehr groß sein können, erscheint eine ungefähre Verdreifachung bei unverändertem Notenbild (Achtel als schnellste Werte) unwahrscheinlich hoch. Bei Straube nun ist der Unterschied tatsächlich sehr groß, indem er auch das Adagiotempo fast halbiert; er schreibt aber auch „Un poco mosso“ (nicht etwa „Un poco più mosso“), stellt also keinen Bezug zum vorangehenden Adagio her.

$\mathrm{Ob}$ in ein und demselben Musikstück, ja ob überhaupt mathematische und metrische Tempobezeichnungen nebeneinander verwendet wurden, ist selbst bei den Anhängern der metrischen Theorie umstritten. Die Theorie eines „variablen Metronomgebrauches“, die bereits von Willem Retze Talsma ${ }^{8}$ vertreten wurde, ging jedenfalls bisher davon aus, dass langsame Tempi mathematisch und schnelle metrisch notiert werden. Hier aber behauptet Stewen das Gegenteil: Reger habe in der Harmoniumfassung das Adagio metrisch und das in „Più mosso" geänderte „Vivace“ mathematisch notiert. Abgesehen von dieser Inkonsequenz, die den „variablen Metronomgebrauch“ noch fragwürdiger macht als er ohnehin schon ist, erscheint es alles andere als plausibel, dass ein Komponist oder Herausgeber den Interpreten mit einem Wechsel von mathematischer zu metrischer Notation innerhalb ein und desselben Musikstückes verwirren wollte. Vielmehr scheint es so, dass Reger zunächst (1908) nur die schnellen Tempi reduziert hat, später (1913) auch die langsamen.

8 Willem Retze Talsma, Wiedergeburt der Klassiker, Bd. 1: Anleitung zur Entmechanisierung der Musik, Innsbruck 1980. 


\section{Fuge.}

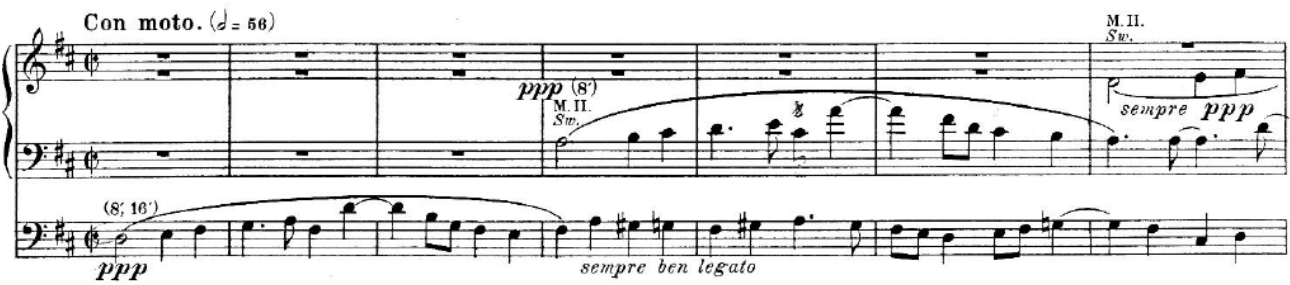

$\mathrm{Nb} 3$ : Fuge op. 59/6 (Ed. Peters 8757)

In der Fuge op. 59/6 gibt Reger nicht weniger als zehn Metronomangaben, die eine stetige Beschleunigung von $\delta=56$ bis $\delta=80$ zeigen, ohne dass sich das Notenbild mit Achteln als schnellsten Werten ändern würde. Straube beginnt mit ${ }^{\dagger}=92$ „Andante tranquillo“. Zunächst ist auch hier schon aufgrund der Vortragsbezeichnung deutlich, dass Straube ein langsameres Tempo als Reger intendiert. Angesichts des Alla-breve-Zeichens könnte die Achtelnote aber ein Druckfehler für eine Viertel sein, zumal in T. 31 • = 72 „Più tranquillo“ folgt und sich auch die weiteren Metronomzahlen auf die Viertel beziehen. Stewen hält dagegen • = 72 für einen Druckfehler und meint, hier müsse eine Achtel stehen. Dann aber wäre Straubes Tempo auch bei metrischer Interpretation von Regers Angabe fast doppelt so langsam, und es träte genau der Fall ein, den Stewen sonst für so ausgeschlossen hält, dass er ihn als Grundlage für seine Hypothese verwendet.

Zwischen diesen beiden Tempoangaben fordert Reger zweimal „un poco stringendo“ und präzisiert das durch zwei weitere Metronomangaben, die bei Straube leider weggelassen sind: $\delta=60$ (T. 17) und $\delta=64$ (T. 22). Wo Straube dann „Più tranquillo“ schreibt, ist Reger bei $\delta=68$ angekommen; bei ihm ist demnach auch der Verlauf der Tempi ein anderer. Sollte es sich bei $C^{\prime}=92$ (die erste Metronomangabe) um keinen Druckfehler handeln, so müsste Straube eine weitaus exzessivere Beschleunigung im Sinn gehabt haben, wohl beinahe eine Tempoverdoppelung, um • = 72 als „ruhiger“ ansehen zu können. Eigenartigerweise ist seine dritte Metronomangabe (entsprechend der sechsten bei Reger) trotz des „Un poco più mosso" mit $•=60$ langsamer als die zweite. Bei Stewens Interpretation der zweiten Angabe als ' $^{-} 72$ wäre zwar eine Beschleunigung vorhanden, diese wäre aber außerordentlich groß.

\section{Fughetta.}

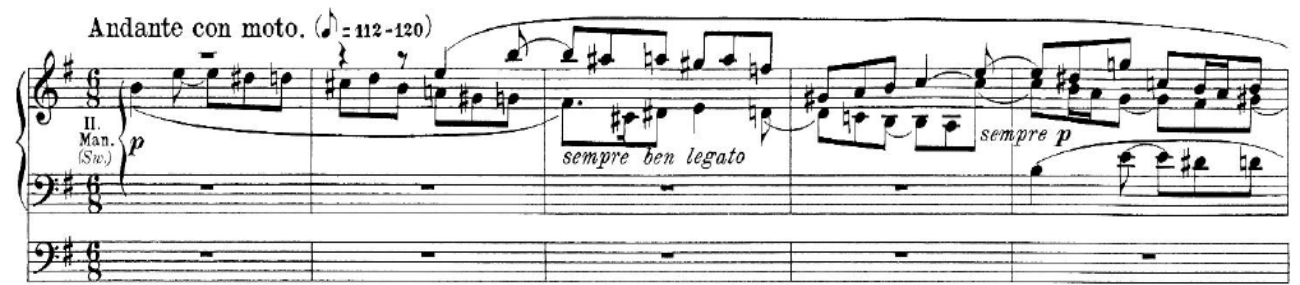

Nb 4: Fughetta op. 80/2 (Ed. Peters 8956)

Die Fughetta op. 80/2 trägt nur eine Tempoangabe: ${ }^{\dagger}=112-120$ bei Reger, ${ }^{\dagger}=60$ bei Straube, der aus Regers „Andante con moto“ ein „Andante tranquillo“ macht, also wiederum eine langsamere Tempoauffassung hat, wobei aber die Halbierung des Tempos weit über diese Modifikation hinauszugehen scheint. 
Zweifache Metronomangaben

Ein weiteres Indiz für metrische Metronomangaben Regers sieht Stewen in zwei Sätzen, bei denen eine zweifache Metronomangabe vorliegt: $\iota^{\dagger}=58-60 / \cdot=26-30$ (richtig wohl 52 statt 58, oder 29 statt 26) im Adagio der Sonate für Klarinette und Klavier op. 107 (1909) sowie $S^{\prime}=25 / .{ }^{A}=50$ in Takt 43 der Bach-Variationen op. 81 (1904). ${ }^{9}$ Damit habe "Reger die Interpreten vor der Hinwendung zum mathematischen Gebrauch des Metronoms gewarnt". ${ }^{10}$ Doch wie sollte ein Interpret eine solche Angabe als Warnung verstehen? Wesentlich naheliegender erscheint der Gedanke, dass man den jeweils langsameren Notenwert als Schlag empfinden soll, während das Metronom den schnelleren Wert angeben muss. Es ist ja auffallend, dass die langsameren Werte jedes Mal unterhalb der Metronomskala liegen.

\section{„Notationsgrundlage“?}

Stewen sieht seine Hypothese auch in einem Aufsatz von Emanuel Gatscher bestätigt, der 1924 schrieb: „Die Regerschen Tempobezeichnungen konnten zu Mißverständnissen führen, die in ihrer katastrophalen Auswirkung kaum auszudenken sind. Straubes Interpretation hat von vornherein Klarheit geschaffen, die Tempofrage führt kaum mehr zu einer folgenschweren Verwirrung, da die Notationsgrundlage erkannt ist."11

Dieser Begriff der „Notationsgrundlage“ soll sich nun auf die Art der Metronomnotation beziehen: metrisch statt mathematisch. Tatsächlich aber kommt der Begriff „Metronom“ in Gatschers Aufsatz überhaupt nicht vor. ${ }^{12}$ In dem zitierten Ausschnitt schreibt er von „Tempobezeichnungen“, und damit sind die Tempowörter, nicht die Metronomangaben gemeint: „Die Tempobezeichnungen Regers, ,vivacissimo“ und ,adagissimo', sind zum Beispiel weniger eine Angabe der ,Schnelligkeit', sondern mehr Ausdrucksbezeichnungen im Sinne einer starken inneren Erregung oder einer starken Entspannung. "13 Der Begriff „Notationsgrundlage“ scheint sich also darauf zu beziehen, was Reger mit den Tempowörtern jeweils meint. Demnach ist Gatschers Aufsatz nicht geeignet, die Hypothese von metrischen Metronomangaben zu stützen. Vielmehr ist anzunehmen, dass der Autor, ein Student Regers und Straubes, einen Wechsel von metrischen zu mathematischen Angaben ausdrücklich thematisiert hätte, da dieser mehr als alles andere zur Klärung der „Tempofrage“ beigetragen hätte.

9 Eine weitere derartige Angabe steht in Nr. 5 der Hiller-Variationen op. 100 (1907): $\delta^{\circ}=96(\bullet=32)$.

10 Stewen, „Metronomzahlen“, S. 2.

11 Emanuel Gatscher, „Einige Bemerkungen zum Studium Regerscher Orgelwerke“, in: Mitteilungen der Max-Reger-Gesellschaft 4 (1924), S. 2-8, dort S. 7.

12 Reger hat nur in zwölf seiner über 30 Opera für Orgel überhaupt Metronomangaben geschrieben, und das oft nur in wenigen der jeweiligen einzelnen Stücke, so dass Metronomzahlen insgesamt eine eher kleine Rolle im Rahmen der Orgelwerke spielen.

13 Ebd. Stewen zitiert diese Stelle auf Englisch (Straube Code, S. 22), ohne zu merken, dass offenbar hier der Schlüssel zur Bedeutung des Begriffes „Notationsgrundlage“ liegt. 
Der historische Kontext

Eine metrische Notation von Pendel- und Metronomangaben ist bisher nirgends nachgewiesen und in vielen Fällen sogar zweifelsfrei widerlegt worden. ${ }^{14} \mathrm{Da}$ ist zum einen der Vergleich mit Takt- und Aufführungsdauerangaben, der die „Metriker“ bis zu der Verstiegenheit gebracht hat, es hätten damals „Doppelsekunden“ existiert oder man hätte nur die Hälfte der Zeit gezählt, so dass z. B. eine Aufführungsdauer von zwei Stunden als „vier Stunden“ gelesen werden müsse; zum anderen ist die Verwendung von Pendel und Metronom in mehreren Quellen eindeutig im heute geläufigen mathematischen Sinn definiert, jedoch in keiner einzigen im metrischen Sinn.

\section{Weitere Metronomangaben Regers}

Ein Beispiel für extrem langsame Metronomangaben aus Regers angeblich „metrischer“ Zeit sind Introduktion, Passacaglia und Fuge für zwei Klaviere op. 96 (1906) mit einem Beginn von.$=64-66$ (Grave assai, 3/4) und einem Ende, wo Reger für die beiden Schlusstakte (Largo) bis auf ${ }^{\prime}=24^{15}$ heruntergeht. Dass dies metrisch gemeint sein sollte, ist kaum vorstellbar. In den Neun Stücken für Orgel op. 129 von 1913 dagegen, also aus einer Zeit, in der Reger Stewen zufolge längst auf mathematische Notation umgestellt hatte, weisen die Metronomangaben, mit Ausnahme der beiden Fugen Nr. 2 und 9, keineswegs eine besondere Langsamkeit auf. Zumindest werden langsame Schlagtempi durch schnelle Notenwerte relativiert:

Nb 5-11: aus Neun Stücke für Orgel op. 129 (2 Hefte), Bote \& Bock 18156 / 18157

\section{Toccata.}

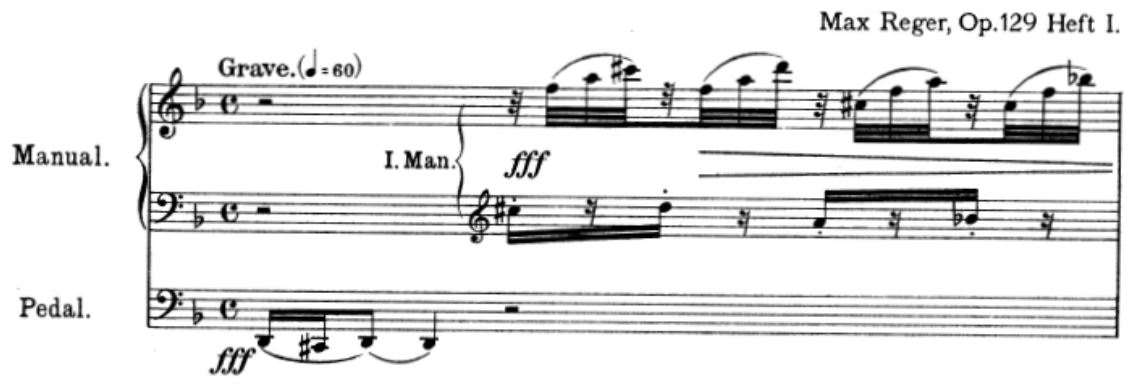

Nb 5: op. 129/1 (Bote \& Bock 18156)

14 Vgl. z. B. Klaus Miehling, Das Tempo in der Musik von Barock und Vorklassik, Wilhelmshaven 1989, 32003, sowie zuletzt dessen Rezension von Lorenz Gadient, Takt und Pendelschlag (= Musikwissenschaftliche Schriften, Bd. 45), München u. Salzburg 2010, in: Concerto 28 (2011), Nr. 238, S. 17.

15 Nicht Viertel, wie bei Hermann Wilske (S. 58) zu lesen ist: Max Reger-Zur Rezeption in seiner Zeit (= Schriftenreihe des Max-Reger-Instituts Bonn, Bd. XI), Wiesbaden, Leipzig u. Paris 1995. 


\section{Kanon.}

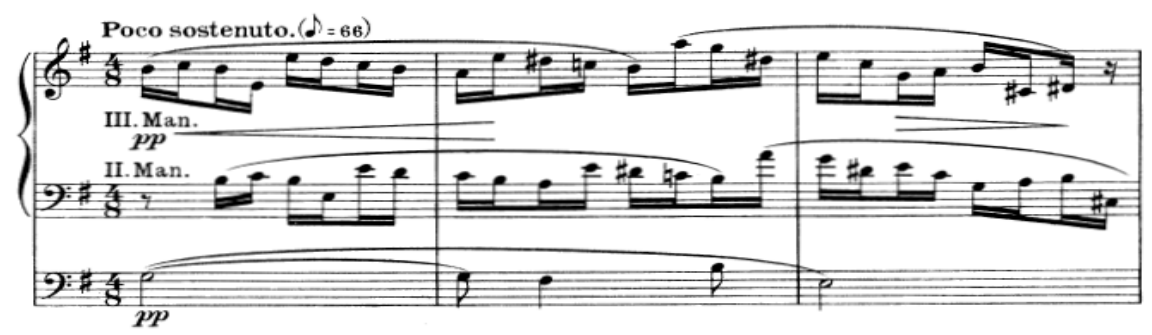

T. 16-18:

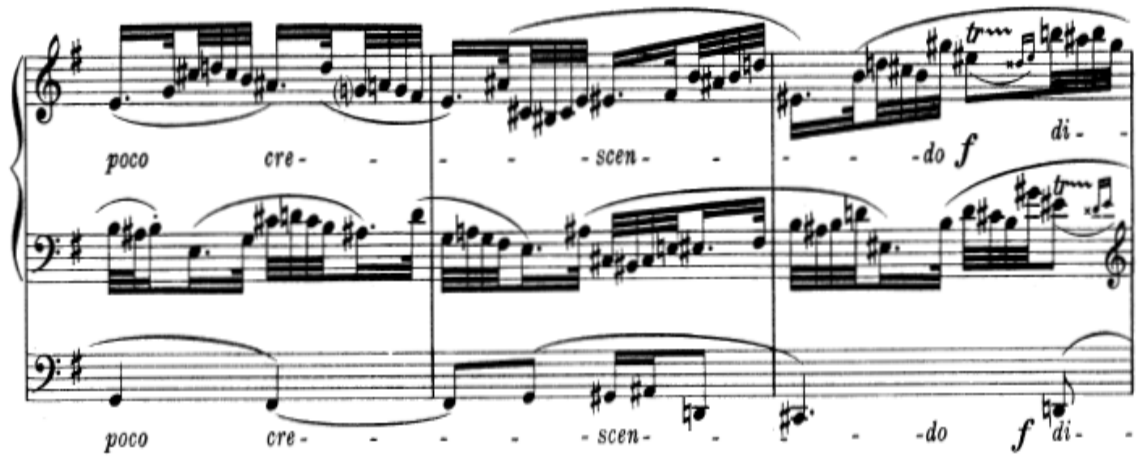

Nb 6: op. 129/3 (Bote \& Bock 18156)

\section{Melodia.}

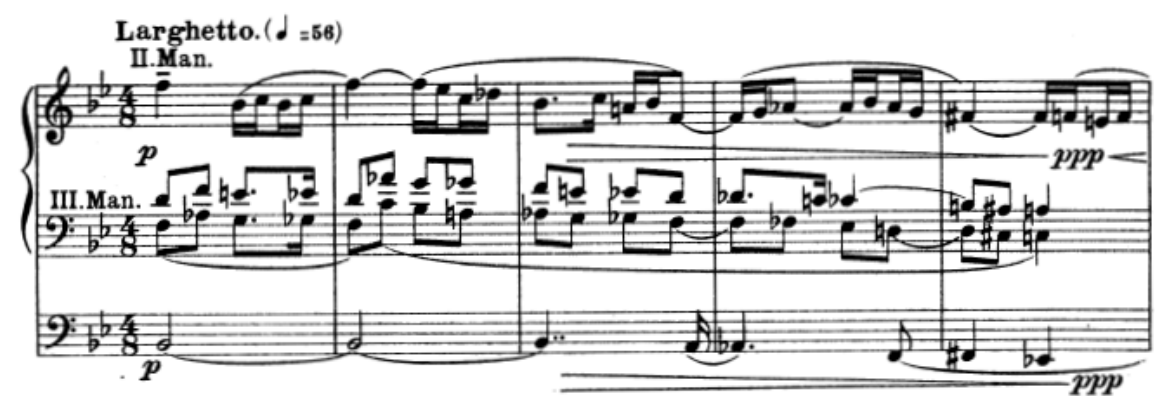

Nb 7: op. 129/4 (Bote \& Bock 18156) 
5. Capriccio.

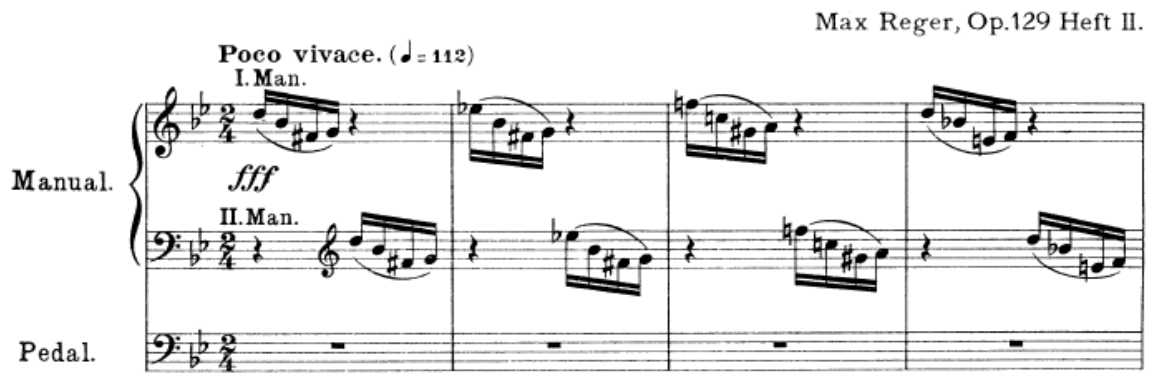

T. 59-62:

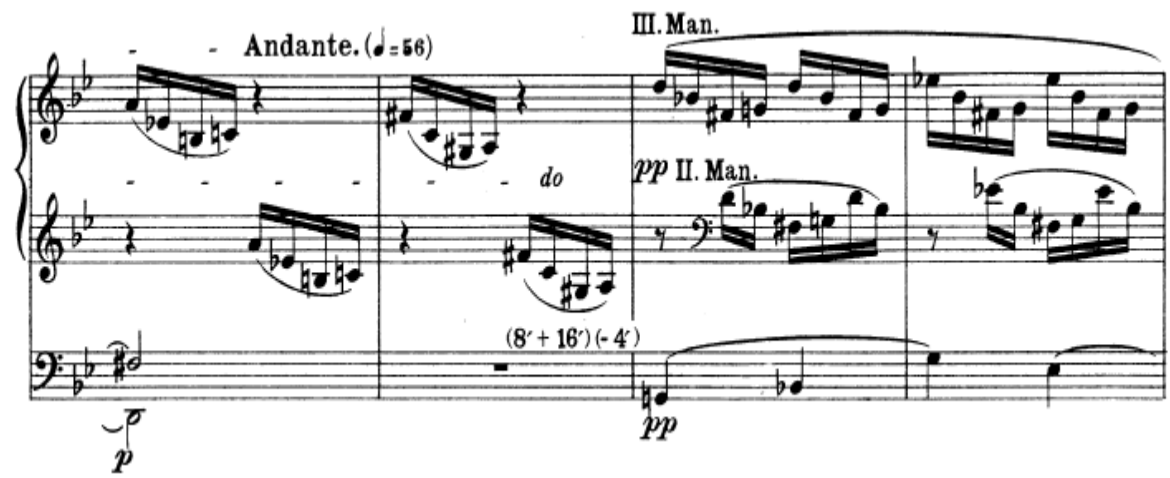

Nb 8: op. 129/4 (Bote \& Bock 18157)

\section{Basso ostinato.}

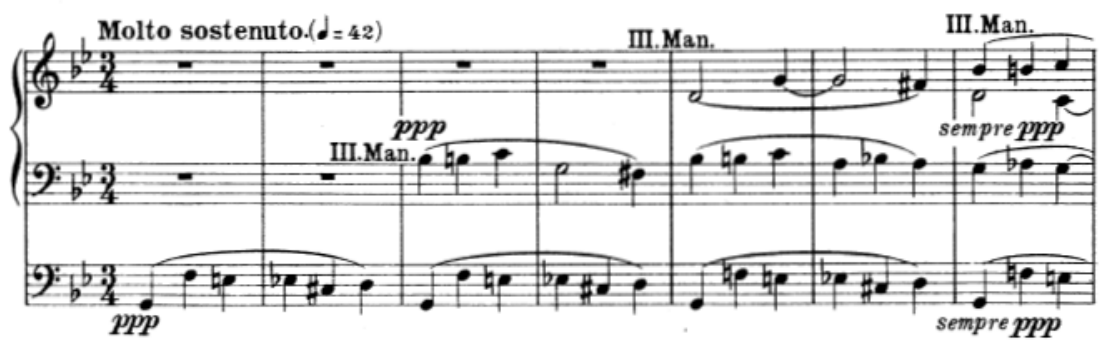


T. 30/31:

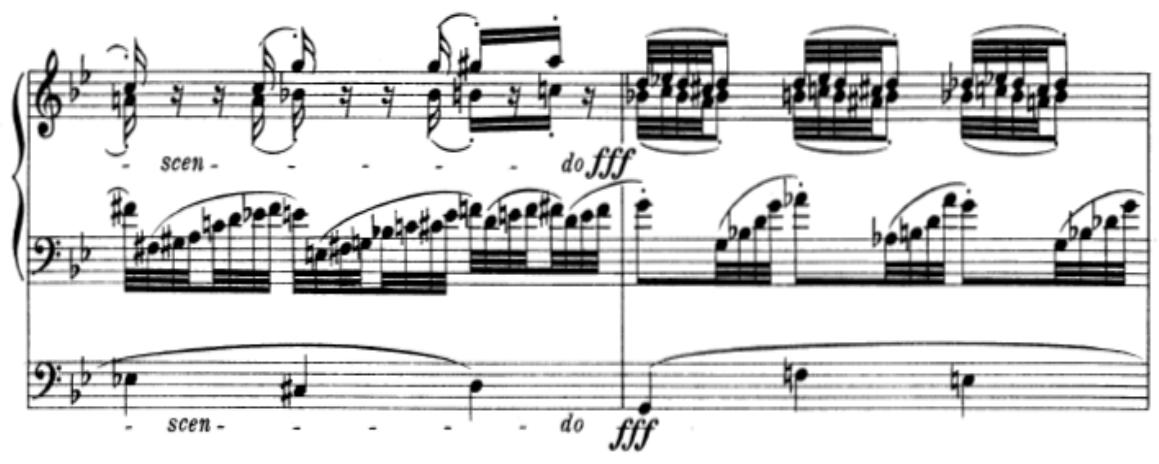

Nb 9: op. 129/6 (Bote \& Bock 18157)

7. Intermezzo.

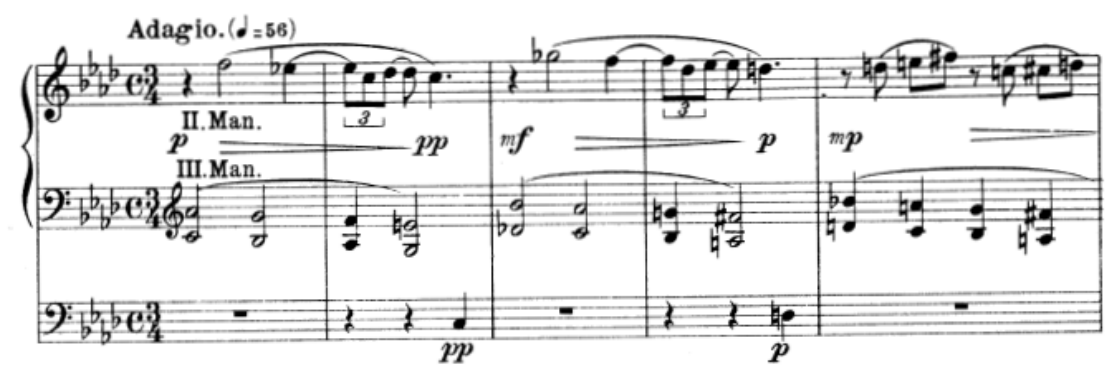

Nb 10: op. 129/7 (Bote \& Bock 18157)

\section{Präludium.}

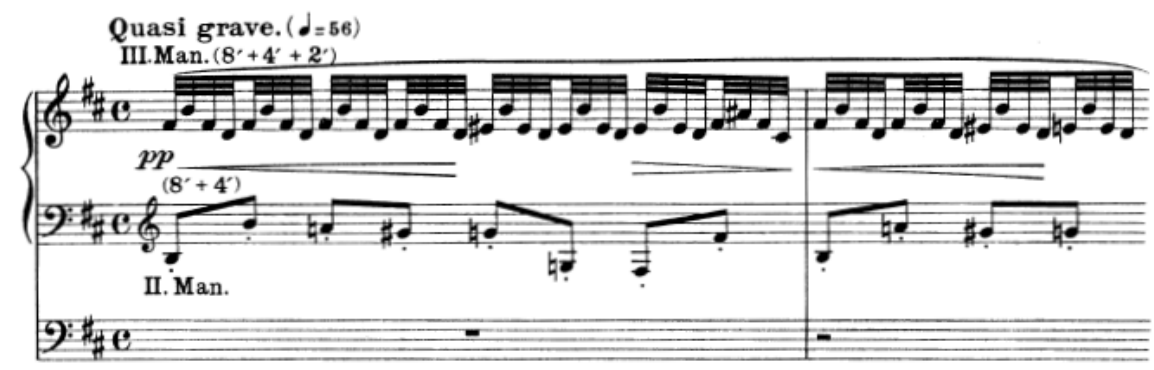

Nb 11: op. 129/8 (Bote \& Bock 18157) 
Die Sichtung eines großen Teiles des Reger'schen Gesamtwerks anhand der in der PetrucciMusikbibliothek (http://imslp.org) zugänglichen Partituren zeigte keine Entwicklung von „schnellen“ (evtl. metrisch zu lesenden) zu „langsamen“ (mathematisch zu lesenden) Tempi.

Regers eigene Interpretationen

Regers Temponahme in der Welte-Mignon-Einspielung des Benedictus op. 59/9, 1913 entstanden, hatten wir bereits betrachtet. Unter den übrigen eingespielten Orgelwerken trägt nur die Melodia op. 59/11 eine Metronomangabe: • = 66-72. Regers Durchschnittstempo liegt bei $\int^{\prime}=43$, beträgt also nur etwa ein Drittel der Vorgabe - das extremste Beispiel unter Regers eigenen Temponahmen. Hier hätte Stewen zwar eine Bestätigung dafür gehabt, dass Reger eigene Orgelwerke in der Hälfte des metronomisch angegebenen Tempos oder gar noch langsamer spielte, doch dessen Interpretationen eigener Klavierwerke, am 8.12.1905 für Welte-Mignon eingespielt, ${ }^{16}$ zeigen ein anderes Bild:

Bezeichnung Takt MM Regers Interpretation

(Durchschnittstempo)

\begin{tabular}{|c|c|c|c|c|}
\hline 6 Intermezzi op. 45, Nr. 5 & Ziemlich schnell & $\begin{array}{l}6 \\
4\end{array}$ & - $120-130$ & .99 \\
\hline $\begin{array}{l}\text { Aus meinem Tagebuch op. 82, } \\
\text { 1. Bd., Nr. } 3\end{array}$ & $\begin{array}{l}\text { Andante sostenuto / Un poco } \\
\text { più moto / a tempo }\end{array}$ & c & . $58 / 68 / 58$ & . $39 / 44 / 33$ \\
\hline dito, 1. Bd., Nr. 5, & Gavotte & $\phi$ & $d 72$ & 62 \\
\hline dito, 1. Bd., Nr. 6 & Sostenuto & $\begin{array}{l}6 \\
8\end{array}$ & .) 56 & D) 49 \\
\hline dito, 1. Bd., Nr. 10 & $\begin{array}{l}\text { Andante innocente / Poco più } \\
\text { mosso / a tempo }\end{array}$ & $\begin{array}{l}3 \\
4\end{array}$ & - $56 / 80 / 56$ & $\cdot 39 / 66 / 33$ \\
\hline dito, 1. Bd., Nr. 11 & Sostenuto ed espressivo & c & $\cdot 62$ & . 40 \\
\hline
\end{tabular}

Wir sehen, dass Reger im Durchschnitt zwar stets langsamer spielt als es seine eigenen Tempoangaben fordern; doch anders als in den oben betrachteten Orgelwerken ist er von einer Halbierung, geschweige denn Drittelung, meist weit entfernt. ${ }^{17}$ In op. 82/I/5 und 6 liegt das langsame Durchschnittstempo sogar nur an Regers ausgiebigen Rubati und Ritardandi, so dass er das vorgeschriebene Tempo ungefähr einhält, wenn die Musik normal voranschreitet.

Die Intermezzi waren 1900 entstanden, Band 1 des Tagebuches um 1904/05. Zu dieser Zeit soll Reger metrisch notiert haben. Seine Interpretation liegt jedoch in der Regel weit näher an den mathematisch verstandenen als an den metrisch verstandenen Tempi. Ganz offensichtlich notierte Reger also schon damals mathematisch. Nun etwa zu behaupten, er hätte nur in Orgelwerken metrisch notiert, müsste erst einmal plausibel gemacht werden.

16 CD Max Reger and Frieda Kwast-Hodapp today playing their 1905/1920 interpretations. Selected works by Reger (= The Welte Mignon Mystery Vol. V), Tacet 152. Hans-W. Schmitz schreibt im Begleittext: „Von der Firma gibt es exakte Angaben, mit welcher Geschwindigkeit das Papierband über den Skalenblock laufen muss. [...] Die gesamte Spielzeit längerer Tonrollen weicht vom Sollwert höchstens um $2 \%$ ab.“

17 Lediglich die Schlussabschnitte von op. 82/I Nr. 3 und 10 liegen im Durchschnitt nur wenige Metronomstriche über dem halben Tempo. 
Die Bedeutung des Tempos für Reger

In mehreren Ausgaben von Klavierwerken hat Reger seine Metronomangaben von vornherein relativiert. Nur ein Beispiel ${ }^{18}$ sei herausgegriffen. Zu den Bach-Variationen op. 81 notiert der Komponist in der zweiten Ausgabe (Bote \& Bock, 1909): „Ich bitte, a 11 e Metronomangaben als nicht strikte bindend anzusehen; doch dürften die Metronomangaben besonders bei den bewegten (schnellen) Variationen und hauptsächlich bei der Fuge, der ein breites Tempo immer gelegen sein wird, als die überhaupt höchst zulässigen Tempi in Bezug auf ,Schnelligkeit' gelten, wenn nicht der Vortrag auf Kosten der Deutlichkeit leiden soll.“ (Hervorh. orig.)

Hier ist interessant, dass die erste Ausgabe (Lauterbach \& Kuhn, 1904) in Regers angeblich „metrische“ Zeit fällt, während die zweite, welche die Fußnote enthält, kurz nach dem angeblichen Wechsel zur mathematischen Notation veröffentlicht wurde. Die Metronomangaben wurden jedoch nicht verändert.

$\mathrm{Zu}$ den mutmaßlichen Gründen, weshalb Reger speziell in Orgelwerken zu schnelle Metronomangaben notiert hat, zählt Karl Matthaei seine „Angst vor dem Schlepptempo vieler Organisten“ sowie die Tatsache, „dass Reger zumeist und namentlich während der Weidener Zeit keine Orgel zum unmittelbaren Ausprobieren seiner Werke zur Verfügung hatte."19

Der zuerst genannte Grund wird durch Roderich von Moysisovics bestätigt, der bereits 1906 im Musikalischen Wochenblatt schrieb, Reger habe ihm gegenüber geäußert, „dass er die raschen Tempobezeichnungen nur zwecks Verhinderung des heutzutage allbeliebten ,Verschleppens" gebe“. ${ }^{20}$ Wenn hier - es ist nach Stewen noch die Zeit der metrischen Notation - von „raschen Tempobezeichnungen“ die Rede ist, dürften sie kaum metrisch gemeint sein.

Weitere Aussagen Regers belegen, dass er eigene Metronomisierungen im Nachhinein als zu schnell empfand. Im Mai 1910 sagte er zum Organisten Gerard Bunk: „Junger Mann, spielens meine Sachen halt net zu schnell; Brahms und ich, mir haben den gleichen Fehler gemacht: mir schrieben unsere Tempi halt viel zu schnell auf, spielens alles recht ruhig, auch wanns schneller dasteht. "21 In einem Brief an den Herzog von Sachsen-Meiningen vom 7.1.1913 rechtfertigte sich Reger für die Missachtung Brahms'scher Tempoangaben: „Der in[n] erlich erregte Schaffende ist eben durch die Erregung verführt zu schnelle Tempi vorzuschreiben. Ich weiß das von mir selbst, dass ich Tempi schon angegeben habe, die ich selbst nachher viel langsamer nehme!“22 In beiden Zitaten spricht Reger von „viel“ zu schnell bzw. „viel“ langsamer, und das zeigt, dass es nicht nur um Nuancen geht, sondern

18 Weitere Beispiele sind Fantasie und Fuge für Orgel op. 46, Sonate für Violoncello und Klavier op. 78, Telemann-Variationen op. 134.

19 1943; zit. n. Christopher Anderson, Max Reger and Karl Straube. Perspectives on an Organ Performing Tradition, Aldershot u. Burlington 2003, S. 181.

20 Zit. n. Alexander Becker et al. (Hrsgg.), Reger-Werkausg., Abt. I, Bd. 2, Stuttgart 2011, begleitende DVD, „Zur Tempowahl“, (3).

21 Zit. n. ebd. Man findet hier weitere Zitate aus Regers Lebzeiten oder den Jahren danach, die sich ähnlich über Regers Metronomangaben äußern - von einer metrischen Lesart, die das „Missverständnis“ aufklären würde, ist niemals die Rede.

22 Hedwig Mueller von Asow u. Erich Hermann Mueller von Asow (Hrsgg.), Max Reger: Briefwechsel mit Herzog Georg II. von Sachsen-Meiningen, Weimar 1949, S. 92 (Hervorh. orig.). Stewen selbst zitiert diese Stelle in englischer Übersetzung (Straube Code, S. 22). 
um sehr große Unterschiede, wie sie eben in Regers Welte-Mignon-Einspielungen für Orgel und in Straubes Metronomangaben dokumentiert sind.

Dass sich Regers Unsicherheit bei der Metronombezeichnung nicht nur auf Orgelund Klavierwerke erstreckte, belegen zahlreiche Berichte von Kammermusikpartnern und Kritikern über Abweichungen Regers, auch als Dirigent, von seinen eigenen Metronomzahlen und von unterschiedlichen Temponahmen derselben Stücke je nach Laune und Tagesform. ${ }^{23}$ Symptomatisch ist eine Konzertkritik im Aachener Anzeiger vom 4. Oktober 1911: „In der Tempinahme läßt er sich ganz von der Empfindung des Augenblicks leiten. "24

Bemerkenswert ist in diesem Zusammenhang auch Regers gelegentlich festzustellende Praxis, Fugen langsam zu beginnen und dann allmählich zu beschleunigen. ${ }^{25}$ Das normalerweise von Anfang bis Ende einheitliche Notenbild einer Fuge und der einheitliche musikalische Charakter ließen ein gleichbleibendes Tempo erwarten. In all diesen Beispielen wird deutlich, dass das Tempo für Reger kein konstitutives Element der Komposition, sondern einen Parameter der Interpretation darstellte, der in weiten Grenzen flexibel war.

\section{Die Rolle von Karl Straube}

Dass Straube außergewöhnlich langsame Tempi bevorzugte, ist mehrfach überliefert und auch in Welte-Mignon-Einspielungen zu hören. Walter Niemann schrieb zur Aufführung von Regers Psalm 100 op. 106 im Juni 1910 unter der Leitung Straubes, dieser neige dazu, „langsame Tempi bis zur Rhythmenlosigkeit zu verschleppen und bewegteren durch sehr gehaltenes Zeitmaß die Kraft zu nehmen“. ${ }^{26}$

Hat Straube Reger hinsichtlich einer Verlangsamung der Tempi beeinflusst? Das ist sehr wahrscheinlich. Reger blieb zeitlebens in einer seltsamen künstlerischen Abhängigkeit von seinem Freund. Wie weit diese ging, zeigt ein Bericht von Regers Ehefrau Elsa, wonach er ein „3/4 fertige[s]“ Requiem für Soli, Chor, Orgel und Orchester ${ }^{27}$ aufgab, nachdem Straube es kritisiert hatte: „Max ist so unsagbar leicht zu beeinflussen u. steht z.Z. wieder ganz unter St[raubes] Bann." 28

Straube selbst bekannte, dass Reger ihm praktisch freie Hand ließ: „Fragen der Interpretation haben wir, was Orgelmusik angeht, wohl nie besprochen. Er war von einer so restlosen Güte zu mir in diesem Punkte, dass darüber zu sprechen mir völlig unmöglich ist." 29

23 Vgl. Otmar Schreiber, Max Reger in seinen Konzerten, Teil 1: Reger konzertiert (= Veröffentlichungen des Max-Reger-Institutes, Bd. 7 Teil 1), Bonn 1981, dort S. 113-116, 152 u. 156; Wilske, Max Reger, S. 65ff; Alexander Becker et al., Reger-Werkausg., Abt. I, Bd. 2, S. XXf u. DVD, „Zur Tempowahl““.

24 Zit. n. Schreiber, Reger konzertiert, S. 114.

25 Vgl. z. B. die oben erwähnte Metronomisierung der Fuge op. 59/6.

26 Leipziger Neueste Nachrichten, 9.6.1910, zit. n. Susanne Popp u. Susanne Shigihara (Hrsgg.): Max Reger: Briefwechsel mit dem Verlag C. F. Peters (= Veröffentlichungen des Max-Reger-Institutes 13), Bonn 1995, S. 411.

27 Die Besetzung geht aus dem Werkverzeichnis in MGG2 hervor (Personenteil, Bd. 13, Sp. 1414). Dort ist nur von Introitus und Dies Irae die Rede; freilich könnte der Rest des „3/4 fertige[n]“ Werkes verschollen sein.

28 Brief vom 19.12.1914 an Fritz Stein., zit. n. Susanne Popp (Hrsg.), Max Reger: Briefe an Karl Straube (= Veröffentlichungen des Max-Reger-Institutes 10), Bonn 1986, S. 246.

29 Bernhard M. Huber, Max Reger - Dokumente eines ästhetischen Wandels, Stuttgart 2008, S. 314, zit. n. Alexander Becker et al., „Zur Tempowahl“ (5). 
Wenn Reger, zu einer Zeit, als er auf der Höhe seines Ruhmes stand, ein weit gediehenes Werk für Chor und Orchester auf Anraten Straubes fallen lässt: Ist dann nicht auch vorstellbar, dass er sich von ihm dazu bewegen ließ, eine große Verlangsamung der Tempi in seinen Orgelwerken zu akzeptieren und schließlich auch selbst auszuführen?

\section{Verlorengegangene Selbstverständlichkeit?}

Stewen meint: „Offensichtlich waren Straubes Änderungen selbstverständlich für Straubes Zeitgenossen, weil keiner sie zu bezweifeln schien und weil Straube es unnötig fand, seine Beweggründe in einem Vorwort oder in Fußnoten zu seinen Editionen zu erklären. “30

Wenn jedoch Reger bis 1907 „selbstverständlich“ - denn es musste ja kein Wort darüber verloren werden - die metrische Notationsweise verwendete, Straube aber nur fünf Jahre später „selbstverständlich“ die mathematische, warum gibt es dann weder aus den Jahren dazwischen, noch aus einer anderen Zeit, irgendeine Quelle, welche diesen Wechsel beschreibt? Ein solcher Wechsel, wenn er denn „selbstverständlich“ gewesen wäre, hätte wie etwa die Festlegung des Kammertons offiziell von einer Tonkünstlervereinigung beschlossen werden müssen, und es gäbe schriftliche Zeugnisse darüber. Existierte aber kein offizieller Beschluss, sondern mehr oder weniger eine Beliebigkeit in der Handhabung, dann hätte zumindest für eine gewisse Übergangszeit in jeder Veröffentlichung, die Metronomzahlen enthält, klargestellt werden müssen, welche Lesart gemeint sei; von einer „Selbstverständlichkeit“ könnte also auch dann keine Rede sein.

Als der Organist Walter Fischer 1910, nur zwei Jahre nach Regers angeblichem Notationswechsel, einen Vortrag „Über die Wiedergabe der Orgelkompositionen Max Regers“ hielt, thematisierte er zwar die Metronomisierungen, die „nur allgemeine Hinweise auf das rechte Tempo“ seien, „aber nicht verbindlich für den Spieler“, 31 verlor jedoch kein Wort über einen Wechsel von metrischer zu mathematischer Notation, der Stewen zufolge gerade zu dieser Zeit von großer Aktualität gewesen wäre.

Ironischerweise haben gerade die Befürworter der metrischen Theorie dadurch, dass sie die Aufmerksamkeit auf die historischen Quellen zum Tempo gelenkt haben, zu der von ihnen abgelehnten Beschleunigung im Bereich der historischen und „historisch informierten “ Aufführungspraxis beigetragen. Dennoch gelingt es ihnen durch rhetorisches Geschick immer wieder, mit ihrem subjektivem Unbehagen gegenüber schnellem Spiel Zweifel zu säen - sogar in der etablierten Musikwissenschaft. ${ }^{32}$ Eine ausführliche Auseinandersetzung mit Stewens Argumenten erschien daher geboten.

30 Stewen, „Metronomangaben“, S. 1.

31 Zit. n. Alexander Becker et al., „Zur Tempowahl“ (3).

32 Ein weiteres Beispiel hierfür, neben der Annahme von Stewens Dissertation, ist die zwar nicht unkritische, aber im Ergebnis doch unsichere Rezension von Gadients Buch Takt und Pendelschlag durch Julian Caskel in Mf65 (2012), S. $271 \mathrm{ff}$. 